\title{
A Marginal Contribution Theory-Based Energy Efficiency Contribution Analysis for Integrated Energy System
}

\author{
Shihai Yang ${ }^{1,2,3}$, Mingming Chen ${ }^{1,3 *}$ and Qiang Zuo ${ }^{1,3}$ \\ ${ }^{1}$ State Grid Jiangsu Electric Power Co. LTD., Nanjing, China, ${ }^{2}$ College of Energy and Electrical Engineering, Hohai University, \\ Nanjing, China, ${ }^{3}$ State Grid Electric Power Demand Side Coordinated Control Technology Joint Laboratory, Nanjing, China
}

The energy efficiency analysis is a prerequisite for the construction of the integrated energy system (IES). In this study, a novel energy efficiency analysis method is proposed considering different energy subsystems in the IES. First, the energy efficiency index of the subsystems and conversion devices is formed for elaborating their influence on the IES. The IES is composed of four energy subsystems, i.e., power/gas/heat/cooling subsystems, and six energy conversion devices. Next, the energy efficiency contribution models of energy subsystems and conversion devices are proposed based on their energy efficiency index, respectively. Then, in order to calculate the energy flow in the IES, an equivalent topological model of the IES weighted directed graph is proposed to calculate the energy efficiency contribution. Finally, an actual park is employed to illustrate the validity of the proposed analysis method.

OPEN ACCESS

Edited by:

Yingjun $W u$,

Hohai University, China

Reviewed by: Bei Han,

Shanghai Jiao Tong University, China

Yujian Ye,

Southeast University, China

*Correspondence:

Mingming Chen

rd1228@163.com

Specialty section:

This article was submitted to Smart Grids,

a section of the journal Frontiers in Energy Research

Received: 11 June 2021

Accepted: 21 July 2021

Published: 25 August 2021

Citation:

Yang S, Chen M and Zuo Q (2021) A Marginal Contribution Theory-Based Energy Efficiency Contribution Analysis for Integrated Energy System. Front. Energy Res. 9:723665. doi: 10.3389/fenrg.2021.723665
Keywords: integrated energy system, weighted directed graph, energy efficiency contribution, energy efficiency, energy quality coefficient

\section{INTRODUCTION}

Energy is the fundamental and motivation of society development. With the rapid growth of the economy, the energy demand has a sharp increase. In the past, the main source for the energy demand is the traditional fossil energy sources, such as coal and oil. However, these fossil energy sources caused a huge concern on the environment pollution.

Therefore, in order to cope with the challenges of environmental pollution and shortage of conventional energy (Zhang et al., 2020), the concept of IES has gained widespread attention (Wang et al., 2017) because it can realize the various energy complementarity (Xue, 2015). In order to achieve the purpose of the IES, it is important to improve the energy utilization efficiency of IES(Abu-Rayash and Dincer, 2020).

The global renewable and sustainable energy industry has witnessed a rapid development over the past decades (Wang et al., 2020). Energy efficiency is a generic index to measure the relationship between the input and output of the energy. There are many energy efficiency assessment methods being proposed and are mainly based on the first law of thermodynamics and the second law of thermodynamics. The first law of thermodynamics focuses on the "quantity" of energy and is mainly studied in the content of heat pumps (Willem et al., 2017), combining cooling, heat, and power (CCHP) units (Wang et al., 2015), HVAC (Alves et al., 2016), and the IES. The second law of thermodynamics focuses on the "quality" of energy. It uses the size of the exergy to evaluate differences in the ability of doing work. The assessment method based on the second law of thermodynamics is frequently utilized in the field of thermodynamic engineering. The first law of thermodynamics ignores the difference of energy grade and the loss of "quality" of the energy in the 
system. While the second law of thermodynamics considers these problems. Therefore, the energy efficiency assessment method used in this paper adopts the second law of thermodynamics.

There are many researches using the assessment method based on thermodynamics to analyze energy systems' energy efficiency. Birol and Keppler (2000) propose that the energy efficiency of the system can be improved from two aspects. The first one introduces new technologies that can increase the productivity of each energy unit, and the second one adjusts the price of energy through economic instruments, but they ignore the reasonable utilization of energy devices which can improve the energy efficiency economically. In Miao et al. (2020), the layering approach has been applied to evaluate the energy efficiency of the IES, and the energy utilization structure of the IES is proposed. While this work does not consider the influence of energy types in the layering approach, Filippini and Hunt (2016); Zou et al. (2013); and Kavousian and Rajagopal (2014) propose a new indicator to evaluate the energy efficiency of the system named energy intensity. The energy efficiency evaluation models considering energy intensity can eliminate the bad factors like unnecessary energy consumption from all production sections of the system. Torres et al. (2018) and Choi et al. (2018) investigate effective ways to improve the energy efficiency of energy storage devices, and the results show that improvements in the energy efficiency of energy storage devices contribute to the availability of the system. Nevertheless, this research ignores the analysis of the operation cost of the energy storage device, which may reduce the energy efficiency of the IES. Liu et al. (2020) propose an improved dynamic energy grading system to evaluate energy in the building. However, this study ignores the analysis of multienergy coupling in the building. Elmirghani et al. (2018) propose some techniques to improve the energy efficiency in general, while this measure ignores the internal energy flow relationship of the evaluation object. Obviously, many studies concentrate on the analysis of system energy efficiency, but paying little attention to the energy grade and difference.

To address these problems, this work proposes the definition of energy efficiency contribution based on the marginal contribution. Actually, "marginal contribution" means "marginal utility". It is a terminology of finance. In microeconomics, the marginal utility means when adding (or removing) a unit of goods or service, the utility increasing (or decreasing) the income of the goods or service. This definition reflects the derivative of the utility to the goods or service. According to this concept, the energy efficiency contribution is represented when the energy efficiency of the energy subsystem or device adds (or removes) a unit, the utility increasing (or decreasing) the energy efficiency of the integrated energy system. Therefore, this article utilizes the partial derivative to define the energy efficiency contribution. First, the structure of the IES is described detailedly and the energy efficiency model of the IES is obtained. Next, according to the definition of energy efficiency, the energy efficiency index of energy subsystems and conversion devices can be obtained. Then, according to the content above, the energy efficiency contribution models of the energy subsystems and devices can be deduced. Finally, this study adopts a weighted directed graph modeling the IES to handle the energy efficiency contribution models. Compared with traditional energy efficiency assessment methods, this method is innovative in that it proposes the concept of energy efficiency contribution according to the different contribution degree of energy subsystems to the energy efficiency of the IES, which reasonably reveals the hierarchy of energy efficiency of energy subsystem.

The rest of this study is structured as follows. Description About Energy Efficiency Indexes of the IES introduces the energy efficiency expression of the IES in detail. In Energy Efficiency Index of Subsystems and Energy Conversion Devices, energy efficiency models of subsystems and energy conversion devices are proposed and illustrated. Then the energy efficiency contribution models of each subsystem and energy conversion devices are provided in Energy Efficiency Contribution Models for Energy Subsystems and Impact of Energy Conversion Devices on the IES. Energy Efficiency Contribution Method Based on Weighted Directed Graph provides a feasible-solution method to the energy efficiency contribution models. Then its simulation analysis on the energy efficiency contribution of each subsystem is provided in Case Study. Some conclusions are finally drawn in Conclusion.

\section{DESCRIPTION OF ENERGY EFFICIENCY INDEXES OF THE IES}

\section{The Structure of the IES}

This article considers four types of energy, i.e., power, heat, cooling, and natural gas. The IES can be divided into the power subsystem, the heat subsystem, the cooling subsystem, the natural gas subsystem, and the energy conversion devices like power boiler, combined heat and power (CHP) unit, power to gas (P2G), gas boiler, and absorption chiller, as shown in Figure 1. Each energy subsystem includes energy transmission, storage, and load. These energy conversion devices include the conversion and transmission of energy, and the loss of transmission is not considered. The natural gas can be converted into heat by the gas boiler. The waste heat recovery boiler in the CHP converts the natural gas into heat, and the gas turbine in the CHP converts the natural gas into power. $\mathrm{P} 2 \mathrm{G}$ can convert the off-peak power into natural gas which is easy to store. In addition, refrigeration equipment can convert the power into cooling or into heat by the power boiler. The absorption chiller can convert the heat into cooling, and it is one of the energy inputs of the cooling subsystem.

This section mainly analyzes the energy efficiency assessment model of the IES, making preparation for the analysis of the energy efficiency of energy subsystems and energy conversion devices.

\section{Energy Efficiency Assessment Model of the IES}

The energy efficiency of the IES can be defined as the ratio of the energy demand to the energy input from the external system. It can reflect the energy loss in the IES. When the system contains energy storage device, it needs to be considered in the process of energy transmission. 


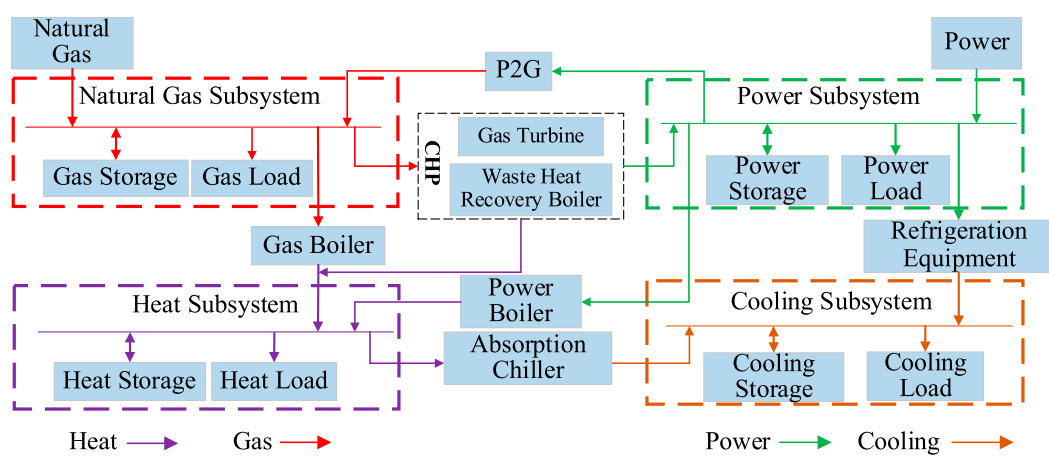

FIGURE 1 | The structure of the integrated energy system.

The traditional calculation method of energy efficiency only considers the quantity superposition of energy. However, the energy system has another important attribute like quality, which has been ignored. The quality of energy means the degree of the energy level. If the energy system ignores the difference in energy level, it cannot represent the degree of energy utilization accurately. As a result, the energy quality loss in the IES cannot be revealed. Therefore, in this research, an energy efficiency assessment model considering the energy quality coefficient is established.

The energy quality coefficient is defined as the ratio of the work did by different energy system to their total energy, which can be shown as

$$
\omega_{e}=\frac{W_{d w}}{W_{t}}
$$

where $W_{d w}$ is the work did by one of the energy systems and $W_{t}$ is the total energy.

The energy quality coefficient reflects the ability of the energy which does work. This ability is usually a fixed value in a standard environment and never vary due to different conversion devices. The detailed formulas for calculating the energy quality coefficient can be found in some papers. Thus, the integrated energy efficiency can be expressed as

$$
\eta_{I E S}^{\prime}=\frac{\sum_{i} l_{i} \lambda_{i}+\sum_{i} \varepsilon_{i} S_{s / i} C_{i}}{\sum_{j} P_{j} C_{j}+\sum_{i}\left(1-\varepsilon_{i}\right) S_{r / i} C_{i}}
$$

where $P_{j}$ denotes the energy input from the external network; $l_{i}$ denotes the load demand for energy $i ; C_{i}, C_{j}$ denotes the energy quality coefficients of energy $i$ and $j ; S_{s / i}, S_{r / i}$ indicates the stored and discharged energy value of energy $i ; \varepsilon_{i}$ indicates the state of the energy storage device, which is 1 when storing energy and 0 when discharging energy.

The energy flow in each subsystem can be clearly seen in Figure 1. Based on the energy flow of all subsystems, the integrated energy efficiency $\eta_{\text {IES }}$ can be expressed as

$$
\eta_{I E S}=\frac{\left(l_{p}+S_{s / p}\right) C_{p}+\left(l_{h}+S_{s / h}\right) C_{h}+\left(l_{c}+S_{s / c}\right) C_{c}+\left(l_{g}+S_{s / g}\right) C_{g}}{\left(P_{p}+S_{r / p}\right) C_{E}+\left(P_{g}+S_{r / g}\right) C_{G}+S_{r / h} C_{H}+S_{r / c} C_{c}}
$$

where $P_{p}, P_{g}$ represent the power and natural gas purchased from the external pipeline network, respectively; $l_{p}, l_{h}, l_{c}, l_{g}$ indicate power, heat, cooling, and gas load, respectively; $S_{s / p}, S_{s / h}, S_{s / \mathcal{c}}, S_{s / g}$ represent the actual energy stored after taking into account the storage loss of the power, heat, cooling, and gas storage device, respectively; $S_{r / p}, S_{r / g}, S_{r / h}, S_{r / c}$ represent the actual energy discharged after taking into account the energy loss of the power, gas, heat, and cooling storage device, respectively; $C_{E}$, $C_{G}, C_{H}$ represent the energy quality coefficient for power, natural gas, and heat energy; $C_{p}, C_{h}, C_{c}, C_{g}$ represent the energy quality coefficient for power, heat, cooling, and air load.

According to the energy flow, the load demand of each subsystem, i.e., $l_{p}, l_{h}, l_{c}, l_{g}$ can be shown as

$$
\begin{gathered}
l_{p}=W_{p}-E_{p-c}-E_{p-h}-E_{p-g}-\gamma_{p} S_{s / p} / \eta_{s / p} \\
l_{h}=W_{h}-H_{h-c}-\gamma_{h} S_{s / h} / \eta_{s / h} \\
l_{c}=W_{c}-\gamma_{c} S_{s / c} / \eta_{s / c} \\
l_{g}=W_{g}-G_{g-h}-G_{c h p}-\gamma_{g} S_{s / g} / \eta_{s / g}
\end{gathered}
$$

where $W_{p}, W_{h}, W_{c}, W_{g}$ denote the supply of power, heat, cooling, and gas; $E_{p-c}, E_{p-h}, E_{p-g}$ represent the power energy consumed to form cooling, heat, and gas, respectively; $H_{h-c}$ indicates the heat energy consumed to form cooling; $G_{g-h}$, $G_{c h p}$ represent the amount of natural gas supplied to the gas boiler, CHP by the natural gas subsystem, respectively; $\gamma_{p}, \gamma_{h}, \gamma_{c}, \gamma_{g}$ indicate the state of the power, heat, cooling, and gas storage device, with 1 for storing and 0 for discharging; $\eta_{s / p}, \eta_{s / h}, \eta_{s / c}, \eta_{s / g}$ indicate the energy storage efficiency of power, heat, cooling, and gas storage device, respectively.

\section{ENERGY EFFICIENCY INDEX OF SUBSYSTEMS AND ENERGY CONVERSION DEVICES}

The energy efficiency represents the ratio of the demand to the supply of the power/natural gas/cooling/heat subsystem. It reflects the energy loss during the transmission and storage process of energy subsystem. Similarly, the efficiency of the energy conversion device reflects the utilization degree of energy. In this section, the energy efficiency model of each 
energy subsystem is conducted according to the formula above. In addition, the efficiency model of the energy conversion device is established as well.

\section{Energy Efficiency Index of Energy Subsystems \\ Power Subsystem}

In the power subsystem, the energy efficiency can be expressed as

$$
\eta_{p}=\frac{W_{p, o u t}}{W_{p, \text { in }}}
$$

where $\eta_{p}$ is the energy efficiency; $W_{p, o u t}$ and $W_{p, \text { in }}$ are the energy output and input of the subsystem, respectively.

The input power is mainly obtained in three ways: it can be purchased from the main grid, obtained by energy conversion device, and discharged by the storage battery:

$$
W_{p, \text { in }}=P_{p}+E_{c h p}+\left(1-\gamma_{p}\right) S_{r / p} / \eta_{r / p}
$$

where $\eta_{r / p}$ is the discharging efficiency of the energy storage battery and $E_{c h p}$ is the power generated by the combined heat and power unit.

The output is usually provided for power load and storage battery; otherwise, it is converted into other energy, i.e., natural gas, heat, and cooling energy:

$$
W_{p, \text { out }}=W_{p}-\gamma_{p} S_{s / p}\left(\frac{1}{\eta_{s / p}}-1\right)
$$

where $W_{p}$ is the total power supply and $\eta_{s / p}$ is the charging efficiency of the battery.

In the power transmission process, it is worth pointing out the problem of the bus voltage level and the line transmission efficiency. Therefore, it needs to consider the bus voltage level and the power loss on the line. The power supply can be expressed in

$$
W_{p}=\left[\left(P_{p}+E_{c h p}\right) \eta_{t}+\left(1-\gamma_{p}\right) S_{r / p}\right] \eta_{l}
$$

where $\eta_{t}$ and $\eta_{l}$ are the efficiency of the translator and power line, respectively.

Substituting $(9,10)$ into $(8)$, the energy efficiency can be written as

$$
\eta_{p}=\frac{W_{p}-\gamma_{p} S_{s / p}\left(\frac{1}{\eta_{s / p}}-1\right)}{P_{p}+E_{c h p}+\left(1-\gamma_{p}\right) S_{r / p} / \eta_{r / p}}
$$

\section{Natural Gas Subsystem}

In the natural gas subsystem, the energy input is mainly from the purchased gas and the gas transferred from the energy conversion devices. The output is provided for gas load and gas storage device and converted into power or heat.

$$
\begin{gathered}
W_{g, \text { in }}=P_{g}+G_{p-g}+\left(1-\gamma_{g}\right) S_{r / g} / \eta_{r / g} \\
W_{g, \text { out }}=W_{g}-\gamma_{g} S_{s / g}\left(\frac{1}{\eta_{s / g}}-1\right)
\end{gathered}
$$

where $\eta_{s / g}$ and $\eta_{r / g}$ are the storage and discharge efficiency of the gas storage device.
The gas supply can be written as

$$
W_{g}=P_{g}+G_{p-g}+\left(1-\gamma_{g}\right) S_{r / g}
$$

where $G_{p-g}$ represents the natural gas generated by power energy through $\mathrm{P} 2 \mathrm{G}$.

Natural gas is a kind of gas energy; there could not be too much loss during the process of gas transmission and storage. The energy efficiency index of the natural gas subsystem is defined as

$$
\eta_{g}=\frac{W_{g}-\gamma_{g} S_{s / g}\left(\frac{1}{\eta_{s / g}}-1\right)}{P_{g}+G_{p-g}+\left(1-\gamma_{g}\right) S_{r / g} / \eta_{r / g}}
$$

\section{Heat Subsystem}

In the heat subsystem, the input is mainly from the heat transferred from the energy conversion devices and the heat discharged by heat energy storage devices. The output is provided for heat load and heat storage device and converted into cooling energy.

$$
\begin{gathered}
W_{h, \text { out }}=W_{h}-\gamma_{h} S_{s / h}\left(\frac{1}{\eta_{s / h}}-1\right) \\
W_{h, \text { in }}=H_{g-h}+H_{c h p-h}+H_{g-h}+\left(1-\gamma_{h}\right) S_{r / h} / \eta_{r / h}
\end{gathered}
$$

where $\eta_{s / h}$ and $\eta_{r / h}$ indicate the efficiency of the heat storage device in storing and discharging heat, respectively.

During the process of heat transmission and storage, there may exist the loss of temperature, which should be taken into consideration. In this article, the energy consumption rate of pipelines is utilized to describe the loss of temperature (Kong et al., 2020). Hence, the heat supply can be expressed as

$$
W_{h}=\left[H_{g-h}+H_{c h p-h}+H_{p-h}+\left(1-\gamma_{h}\right) S_{r / h}\right]\left(1-0.01 l_{h} \sigma_{h}\right)
$$

where $H_{g-h}, H_{c h p-h}$ represent the heat energy produced by natural gas through gas boiler and waste heat recovery boiler, respectively; $H_{p-h}$ indicates the heat energy generated by power through power boiler; $l_{h}$ indicates the length of the heat pipe network; $\sigma_{h}$ represents the dissipation rate per $100 \mathrm{~m}$ of the heat pipe network.

The energy efficiency index of the heat subsystem is defined as

$$
\eta_{h}=\frac{W_{h}-\gamma_{h} S_{s / h}\left(\frac{1}{\eta_{s / h}}-1\right)}{H_{g-h}+H_{c h p-h}+H_{p-h}+\left(1-\gamma_{h}\right) S_{r / h} / \eta_{r / h}}
$$

\section{Cooling Subsystem}

In the cooling subsystem, the energy input is mainly from the cooling energy transferred from the energy conversion devices and the cooling energy discharged by the cooling storage device. The output is provided for the cooling load, cooling storage device.

$$
\begin{gathered}
W_{c, \text { out }}=W_{c}-\gamma_{c} S_{s / c}\left(\frac{1}{\eta_{s / c}}-1\right) \\
W_{c, \text { in }}=C_{p-c}+C_{h-c}+\left(1-\gamma_{c}\right) S_{r / c} / \eta_{r / c}
\end{gathered}
$$


where $\eta_{s / c}$ and $\eta_{r / c}$ indicate the efficiency of the storage and discharge of the cooling storage device.

Similar to the heat subsystem, there also exists the loss of temperature during the process of energy transmission and storage. The total supply quantity of cooling energy considering the energy consumption rate can be expressed as

$$
W_{c}=\left[C_{p-c}+C_{h-c}+\left(1-\gamma_{c}\right) S_{r / c}\right]\left(1-0.01 l_{c} \sigma_{c}\right)
$$

where $C_{p-c}$ indicates the cooling energy converted by refrigeration equipment; $C_{h-c}$ indicates the cooling energy converted by absorption chiller; $l_{c}$ is the length of the cool pipe network; $\sigma_{c}$ indicates the dissipation rate per $100 \mathrm{~m}$ of the cool pipe network.

The energy efficiency index of the cooling subsystem is defined as

$$
\eta_{c}=\frac{W_{c}-\gamma_{c} S_{s / c}\left(\frac{1}{\eta_{s / c}}-1\right)}{C_{p-c}+C_{h-c}+\left(1-\gamma_{c}\right) S_{r / c} / \eta_{r / c}}
$$

\section{Efficiency Index of Energy Conversion Devices}

In the IES, the energy conversion devices play a necessary role in making a close relationship among the subsystem above. Therefore, the efficiency index of the energy conversion devices should be considered to analyze the energy efficiency of the IES objectively. In this subsection, efficiency indexes of six energy conversion devices are given as follows.

\section{1) Power-to-Heat Device}

In this study, the power-to-heat device is considered as the power boiler. According to the concept of the energy quality coefficient in Description About Energy Efficiency Indexes of the $I E S$, the efficiency index of the power boiler can be written as

$$
\eta_{p-h}=\frac{C_{h}}{C_{E}} \lambda_{c o p}(p-h)
$$

where $\lambda_{c o p(p-h)}$ is the heating coefficient.

2) Power-to-Cool Device

The power-to-cool device is usually considered as refrigeration equipment. The efficiency index of the refrigeration equipment is

$$
\eta_{p-c}=\frac{C_{c}}{C_{E}} \lambda_{c o p(p-c)}
$$

where $\lambda_{c o p(p-c)}$ is the cooling factor for the power-to-cool device.

\section{3) Combined Heat and Power Unit}

The combined heat and power unit is composed of the gas turbine and the waste heat recovery boiler. The gas turbine can convert the natural gas into power and heat, and the waste heat recovery boiler can reutilize the waste heat which is generated in the process of energy conversion. Hence, the efficiency index of the combined heat and power unit is

$$
\eta_{c h p}=\frac{C_{E} E_{c h p}+C_{s} H_{c h p-h}}{C_{G} G_{c h p}}
$$

where $C_{s}$ is the energy quality coefficient of hot steam.

\section{4) Device Converting Natural Gas to Heat}

Besides the combined heat and power unit, the gas boiler is also an efficient device converting gas to heat and its efficiency index can be given as

$$
\eta_{g-h}=\frac{C_{h}}{C_{G}} \lambda_{c o p}(g-h)
$$

where $\lambda_{c o p(g-h)}$ is the heat production coefficient of the gas boiler.

5) Heat-to-Cool Device

The heat-to-cool device in the IES is usually considered as the absorption chiller. The efficiency index of the absorption chiller is

$$
\eta_{h-c}=\frac{C_{c}}{C_{H}} \lambda_{c o p(h-c)}
$$

where $\lambda_{c o p}(h-c)$ indicates the heat-to-cool coefficient of an absorption chiller.

6) P2G Device

The efficiency index of the P2G device is

$$
\eta_{p-g}=\frac{C_{g}}{C_{E}} \lambda_{c o p}(p-g)
$$

where $\lambda_{c o p(p-g)}$ denotes the coefficient of the power-to-gas of P2G.

\section{ENERGY EFFICIENCY CONTRIBUTION MODELS FOR ENERGY SUBSYSTEMS}

The energy efficiency of the IES is determined by that of each subsystem. However, due to the fact that each subsystem has different influence on the efficiency of IES, it is hard to measure the contribution of subsystems to the IES. Therefore, in this section, an energy efficiency contribution model for subsystem is proposed. The IES operator can classify the subsystems according to their contributions. When the energy efficiency of IES decreases, the IES operator should adjust the subsystem whose contribution is high to enhance the efficiency of the IES.

Actually, the contribution degree in this research means marginal utility. It reflects the variation degree of the energy efficiency at one point of time. Therefore, this study uses partial derivative to express the energy efficiency contribution. Thus, the contributions of subsystems can be defined as the partial derivatives demanded by $\eta_{I E S}$ on $\eta_{p}, \eta_{g}, \eta_{h}$, and $\eta_{c}$. We use $\zeta$ to represent the energy efficiency contribution. We use energy efficiency contribution to represent the energy efficiency contribution degree of the energy subsystems and 
conversion devices to the integrated energy system, and the positive or negative of this value indicates whether the influence of the energy subsystem and the energy conversion devices to the integrated energy system is positive or negative. The positive correlation represents that the larger the energy efficiency contribution of the subsystems and devices is, the more obvious the integrated energy efficiency increases. The negative correlation represents that the larger the energy efficiency contribution of the subsystems and devices is, the more obvious the integrated energy efficiency decreases.

\section{Energy Efficiency Contribution of the Power Subsystem}

According to Eqs 2, 3, 12, the energy efficiency contribution of the power subsystem can be obtained as

$$
\begin{aligned}
\zeta_{p}= & C_{p}\left(P_{p}+E_{c h p}+\left(1-\gamma_{p}\right) S_{r / p} / \eta_{r / p}\right)\left[\left(P_{p}+S_{r / p}\right) C_{E}\right. \\
& \left.+\left(P_{g}+S_{r / g}\right) C_{G}+S_{r / h} C_{H}+S_{r / c} C_{c}\right]^{-1}
\end{aligned}
$$

where is the partial derivative demanded by $\eta_{\text {IES }}$ on $\eta_{p} \cdot \gamma_{p}$ indicates the operating state of the energy storage device, which is 1 when storing energy and 0 when discharging energy.

When the energy storage device is in the storage state, the energy efficiency contribution of the power subsystem is

$\zeta_{p}=C_{p}\left(P_{p}+E_{c h p}\right)\left[\left(P_{p}+S_{r / p}\right) C_{E}+\left(P_{g}+S_{r / g}\right) C_{G}+S_{r / h} C_{H}+S_{r / c} C_{c}\right]^{-1}$

And when the energy storage device is in the discharge state, the energy efficiency contribution can be obtained as

$$
\begin{aligned}
\zeta_{p}= & C_{p}\left(P_{p}+E_{c h p}\right. \\
& \left.+S_{r / p} / \eta_{r / p}\right)\left[\left(P_{p}+S_{r / p}\right) C_{E}+\left(P_{g}+S_{r / g}\right) C_{G}+S_{r / h} C_{H}+S_{r / c} C_{c}\right]^{-1}
\end{aligned}
$$

These formulas represent different expressions of the formula under different working conditions of the energy storage device.

\section{Energy Efficiency Contribution of Natural Gas Subsystem}

Similarly, the energy efficiency contribution of the natural gas subsystem can be shown as

$$
\begin{aligned}
\zeta_{g}= & C_{g}\left(P_{g}+G_{p-g}+\left(1-\gamma_{g}\right) S_{r / g} / \eta_{r / g}\right)\left[\left(P_{p}+S_{r / p}\right) C_{E}\right. \\
& \left.+\left(P_{g}+S_{r / g}\right) C_{G}+S_{r / h} C_{H}+S_{r / c} C_{c}\right]^{-1}
\end{aligned}
$$

where is the partial derivative demanded by $\eta_{I E S}$ on $\eta_{g} \cdot \gamma_{g}$ indicates the operating state of the energy storage device, which is 1 when storing energy and 0 when discharging energy.

When the energy storage device is in storing, i.e., $\gamma_{g}=1$, the energy efficiency contribution is

$$
\zeta_{g}=C_{g}\left(P_{g}+G_{p-g}\right)\left[\left(P_{p}+S_{r / p}\right) C_{E}+\left(P_{g}+S_{r / g}\right) C_{G}+S_{r / h} C_{H}+S_{r / c} C_{c}\right]^{-1}
$$

When $\gamma_{g}=0$, the energy efficiency contribution is

$$
\begin{aligned}
\zeta_{g}= & C_{g}\left(P_{g}+G_{p-g}+S_{r / g} / \eta_{r / g}\right)\left[\left(P_{p}+S_{r / p}\right) C_{E}+\left(P_{g}+S_{r / g}\right) C_{G}\right. \\
& \left.+S_{r / h} C_{H}+S_{r / c} C_{c}\right]^{-1}
\end{aligned}
$$

These formulas represent different expressions of the formula under different working conditions of the energy storage device.

\section{Energy Efficiency Contribution of Heat Subsystem}

The energy efficiency contribution of the heat subsystem can be obtained as

$$
\begin{aligned}
\zeta_{h}= & C_{h}\left(H_{g-h}+H_{c h p-h}+H_{p-h}+\left(1-\gamma_{h}\right) S_{r / h} / \eta_{r / h}\right)\left[\left(P_{p}+S_{r / p}\right) C_{E}\right. \\
& \left.+\left(P_{g}+S_{r / g}\right) C_{G}+S_{r / h} C_{H}+S_{r / c} C_{c}\right]^{-1}
\end{aligned}
$$

where the partial derivative demanded by $\eta_{I E S}$ is on $\eta_{h} \cdot \gamma_{h}$ indicates the operating state of the energy storage device, which is 1 when storing energy and 0 when discharging energy.

When $\gamma_{h}=1$, the energy efficiency contribution is

$\zeta_{h}=C_{h}\left(H_{g-h}+H_{c h p-h}+H_{p-h}\right)\left[\left(P_{p}+S_{r / p}\right) C_{E}+\left(P_{g}+S_{r / g}\right) C_{G}+S_{r / h} C_{H}+S_{r / c} C_{c}\right]^{-1}$

When $\gamma_{h}=0$, the energy efficiency contribution is

$$
\begin{aligned}
\zeta_{h}= & C_{h}\left(H_{g-h}+H_{c h p-h}+H_{p-h}+S_{r / h} / \eta_{r / h}\right)\left[\left(P_{p}+S_{r / p}\right) C_{E}\right. \\
& \left.+\left(P_{g}+S_{r / g}\right) C_{G}+S_{r / h} C_{H}+S_{r / c} C_{c}\right]^{-1}
\end{aligned}
$$

These formulas represent different expressions of the formula under different working conditions of the energy storage device.

\section{Energy Efficiency Contribution of Cooling Subsystem}

The energy efficiency contribution of the cooling subsystem can be obtained as

$\zeta_{c}=C_{c}\left(C_{p-c}+C_{h-c}\right.$

$$
\left.+\left(1-\gamma_{c}\right) S_{r / c} / \eta_{r / c}\right)\left[\left(P_{p}+S_{r / p}\right) C_{E}+\left(P_{g}+S_{r / g}\right) C_{G}+S_{r / h} C_{H}+S_{r / c} C_{c}\right]^{-1}
$$

where is the partial derivative demanded by $\eta_{\text {IES }}$ on $\eta_{c} \cdot \gamma_{c}$ indicates the operating state of the energy storage device, which is 1 when storing energy and 0 when discharging energy.

When $\gamma_{c}=1$, the energy efficiency contribution is

$$
\begin{aligned}
\zeta_{c}= & C_{c}\left(C_{p-c}\right. \\
& \left.+C_{h-c}\right)\left[\left(P_{p}+S_{r / p}\right) C_{E}+\left(P_{g}+S_{r / g}\right) C_{G}+S_{r / h} C_{H}+S_{r / c} C_{c}\right]^{-1}
\end{aligned}
$$

When $\gamma_{c}=0$, the energy efficiency contribution is

$$
\begin{aligned}
\zeta_{c}= & C_{c}\left(C_{p-c}+C_{h-c}\right. \\
& \left.+S_{r / c} / \eta_{r / c}\right)\left[\left(P_{p}+S_{r / p}\right) C_{E}+\left(P_{g}+S_{r / g}\right) C_{G}+S_{r / h} C_{H}+S_{r / c} C_{c}\right]^{-1}
\end{aligned}
$$

In conclusion, the energy efficiency contributions above are positively correlated to the energy efficiency of the IES. 


\section{IMPACT OF ENERGY CONVERSION DEVICES ON THE IES}

For the energy conversion devices among energy subsystems in this study, it is necessary to analyze the impact of energy conversion devices on the energy efficiency of the IES. The energy conversion devices influence the flexibility of the system and play a key role in the energy efficiency of the IES. In this article, the energy conversion devices include CHP unit, gas boiler, power boiler, absorption chiller, P2G device, and refrigeration equipment. Similar to the contribution of the subsystems in Energy Efficiency Contribution Models for Energy Subsystems, the contribution of each energy conversion device to the IES can be defined as the partial derivatives demanded by $\eta_{I E S}$ on $\eta_{p-h}, \eta_{p-c}, \eta_{c h p}, \eta_{g-h}, \eta_{h-c}$, and $\eta_{p-g}$. We use $\zeta_{p-h}, \zeta_{p-c}, \zeta_{c h p}, \zeta_{g-h}, \zeta_{h-c}$ and $\zeta_{p-g}$ to represent the energy efficiency contribution of the energy conversion devices.

1) Power-to-Heat Device

According to Eqs 3, 25, the contribution of power boilers can be written as

$\zeta_{p-h}=C_{E}\left(l_{h}+S_{s / h}\right)\left[\lambda_{c o p(p-h)}\left(\left(P_{p}+S_{r / p}\right) C_{E}+\left(P_{g}+S_{r / g}\right) C_{G}+S_{r / h} \lambda_{H}+S_{r / c} C_{c}\right)\right]^{-1}$

where the partial derivative is demanded by $\eta_{I E S}$ on $\eta_{p-h}$. We use this formula to represent the energy efficiency contribution degree of the power boiler.

2) Power-to-Cool Device

According to Eqs 3, 26, the contribution of the refrigeration equipment is

$\zeta_{p-c}=C_{E}\left(l_{c}+S_{s / c}\right)\left[\lambda_{c o p(p-c)}\left(\left(P_{p}+S_{r / p}\right) C_{E}+\left(P_{g}+S_{r / g}\right) C_{G}+S_{r / h} \lambda_{H}+S_{r / c} C_{c}\right)\right]^{-1}$

where the partial derivative is demanded by $\eta_{I E S}$ on $\eta_{p-c}$. We use this formula to represent the energy efficiency contribution degree of the refrigeration equipment.

\section{3) Combined Heat and Power Unit}

The CHP unit consists of a gas turbine and a waste heat recovery boiler, and its impact on energy efficiency is also determined by these devices. According to Eqs 3, 27, the contribution of CHP unit can be obtained as

$\zeta_{c h p}=\frac{\left(l_{p}+S_{s / p}\right) C_{p}+\left(l_{h}+S_{s / h}\right) C_{h}+\left(l_{c}+S_{s / c}\right) C_{c}+\left(l_{g}+S_{s / g}\right) C_{g}}{\left(P_{p}+S_{r / p}\right) C_{E}+\left(P_{g}+S_{r / g}\right)\left(C_{E} E_{c h p}+C_{s} H_{c h p-h}\right)+S_{r / h} \lambda_{H}+S_{r / c} C_{c}} G_{c h p}$

where the partial derivative is demanded by $\eta_{I E S}$ on $\eta_{c h p}$. We use this formula to represent the energy efficiency contribution degree of the combined heat and power unit.

4) Gas-to-Heat Device
According to Eqs 3, 28, the contribution of gas boiler can be written as

$$
\begin{aligned}
\zeta_{g-h}= & C_{G} \\
& \left(l_{h}+S_{s / h}\right)\left[\lambda_{c o p(g-h)}\left(\left(P_{p}+S_{r / p}\right) C_{E}+\left(P_{g}+S_{r / g}\right) C_{G}+S_{r / h} \lambda_{H}+S_{r / c} C_{c}\right)\right]^{-1}
\end{aligned}
$$

where the partial derivative is demanded by $\eta_{I E S}$ on $\eta_{g-h}$. We use this formula to represent the energy efficiency contribution degree of the gas boiler.

\section{5) Heat-to-Cool Device}

According to Eqs 3, 29, the contribution of the absorption chiller is

$\zeta_{h-c}=C_{H}\left(l_{c}+S_{s / c}\right)\left[\lambda_{c o p(h-c)}\left(\left(P_{p}+S_{r / p}\right) C_{E}+\left(P_{g}+S_{r / g}\right) C_{G}+S_{r / h} \lambda_{H}+S_{r / c} C_{c}\right)\right]^{-1}$

where the partial derivative is demanded by $\eta_{I E S}$ on $\eta_{h-c}$. We use this formula to represent the energy efficiency contribution degree of the absorption chiller.

6) Power-to-Gas Device

According to Eqs 3, 30, the contribution of the P2G device can be written as

$\zeta_{p-g}=C_{E}\left(l_{g}+S_{s / g}\right)\left[\lambda_{c o p(p-g)}\left(\left(P_{p}+S_{r / p}\right) C_{E}+\left(P_{g}+S_{r / g}\right) C_{G}+S_{r / h} \lambda_{H}+S_{r / c} C_{c}\right)\right]^{-1}$

where the partial derivative is demanded by $\eta_{I E S}$ on $\eta_{p-g}$. We use this formula to represent the energy efficiency contribution degree of the P2G.

\section{ENERGY EFFICIENCY CONTRIBUTION METHOD BASED ON WEIGHTED DIRECTED GRAPH}

This section proposes an energy efficiency contribution solution method based on a weighted directed graph (Qin et al., 2021). From the above equations, it can be seen that the solution of the model depends on the input and output energy flows, while the physical properties of the integrated energy flows can be simplified by means of a directed graph, establishing a description of the steady-state energy flow relationship of the system.

A directed graph includes the set of nodes $T$ as well as the set of branches $L$; we represent it by $D(T, L)$. The node sets are used to describe the input, output, conversion, and storage nodes of energy within the system, while the branch set represents the connection between nodes. The direction from node $i$ to node $j$ is denoted by $e(i, j)$, and the energy transferred from node $i$ to node $j$ is denoted by $e_{i j}$. In addition, weights are assigned to the branches to characterize the energy loss between the nodes, and the size of the weights indirectly reflects the length of the branch. As shown 


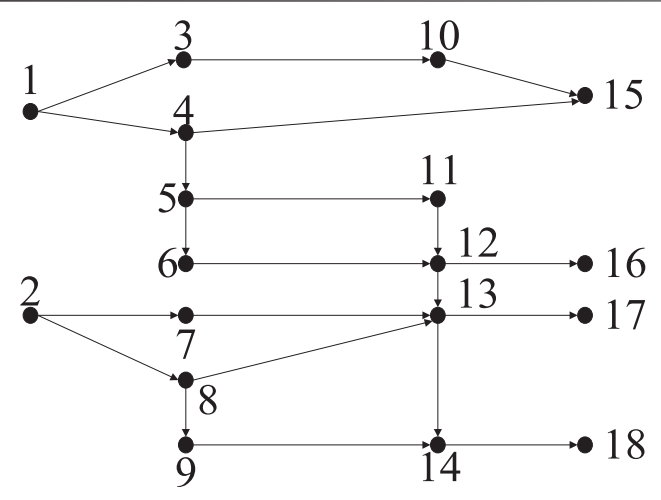

FIGURE 2 | The weighted directed graph of the IES.

in Figure 2, nodes 1 and 2 represent the purchased natural gas and power energy, respectively, and nodes 15, 16, 17, and 18 represent the energy demand side of the system.

According to the definition of a weighted directed graph, the system can be described in terms of a normalized node matrix (Wang et al., 2019). Supposing that there are $N$ nodes and $B$ branches organized, the node-branch incidence matrix (Li et al., 2019) $A=\left(\mathrm{a}_{i k}\right)_{N \times B}$ is a $N \times B$ matrix; i.e.,

$$
a_{i k}= \begin{cases}1, & \text { if branch } l_{k} \text { starts from node } i \\ -1 & \text { if branch } l_{k} \text { ends with node } i \\ 0, & \text { if branch } l_{k} \text { is not connected with node } i\end{cases}
$$

where $l_{k}$ denotes the $k$-th edge, $k=1,2, \cdots, m$.

Considering the value of the weight on each branch, it is necessary to create the weight matrix and define the weight on the branch as

$$
\eta_{i j}= \begin{cases}-\ln \mu_{i j}, & e(i, j) \in L \\ 0, & e(i, j) \notin L\end{cases}
$$

where $\mu_{i j}$ is the energy conversion efficiency between the two nodes.

This article defines $S$ as a path from an input node to an output node. $X=\left(x_{1}, x_{2}, \cdots, x_{\mathrm{m}}\right)^{T}$ represents the connection relationship between nodes. The elements in $X$ are all binary variables, as shown in

$$
x_{k}=\left\{\begin{array}{l}
1, e_{k} \in S \\
0, e_{k} \notin S
\end{array}, k=1,2, \cdots, m\right.
$$

where $e_{k}$ denotes the $k$-th edge, and the length of the path $S$ can be calculated as

$$
d_{s}=\sum_{k} \eta_{i j} x_{k}
$$

Based on the weighted directed graph, the energy flow value required from the starting point can be calculated by the length of the path considering the end node needs per unit of energy flow. It can also obtain the energy flow of all nodes in the path to calculate the energy efficiency of the system and the energy conversion devices and then analyze their contribution.

We use the path length of the weighted directed graph to calculate the energy flow from which the starting point needs supply, when the end exports unit energy flow. According to the weighted directed graph model, we can adopt a discretized energy flow calculation method to handle the energy efficiency model. The specific solution steps can be seen in Figure 3.

1) Initialize the energy flow value of each side in the system weighted directed graph model, and calculate the initial value of the input energy flow value and output energy flow value of the system. Meanwhile, initializing two weight matrices of the system. This step is used to handle some presets of the system.

2) Discretize the energy flow of each output node known by the system into a number of unit step lengths. If a certain energy flow value $\Delta p$ is used as the step length, the output energy flow of the system shown in Figure $\mathbf{2}$ is discretized, respectively.

3) Find a feasible path from each input node to each output node to form the path set of the system. In Figure 2, all available paths from input node 1 to output node 15 can be obtained by solving Eq. 52. After obtaining the path set of the system, the system paths will be sorted according to certain priority rules.

4) The priority path is selected according to the priority order, and the current path length $d_{l}$ of the system is calculated using formula (52) to obtain the increment $e^{d l} \Delta p$ of the input node energy flow value of the system in the unit step length. At the same time, the energy flow value increment of each side in the path is traced. Based on the previous calculation of the energy flow value, superimpose the new calculated value and judge whether the capacity of the side is exceeded. If the capacity of the edge is exceeded, the path will be deleted from the path set.

5) Judge whether the output node of the system has reached the expected energy output demand. If the system demand has been met, all paths ending at the node will be deleted from the path set.

6) According to the calculation results of the energy flow value of each side, update the weight matrix and the path set in the system.

7) Circulate until the system path set is empty or the energy flow of each output node reaches the setting value and complete the energy flow calculation of the system.

8) According to the input and output energy flow values, the energy efficiency contribution models will be calculated.

\section{CASE STUDY}

\section{System Description}

The case study does not analyze the energy efficiency contribution of the energy conversion devices singly, because the content has included the study of the energy conversion devices efficiency in the process of simulating the energy efficiency contribution of the energy subsystem. And the energy flow in the energy subsystems can be calculated by the analysis of the energy conversion devices. As we all know, the energy efficiency contribution of the devices to the integrated energy system is very small. The energy loss of the energy transmission, storage, and supply in the energy subsystem is the main reason of influencing the system energy efficiency. Therefore, we simulate the energy efficiency contribution of the energy subsystem mainly and do not analyze the energy conversion devices. 


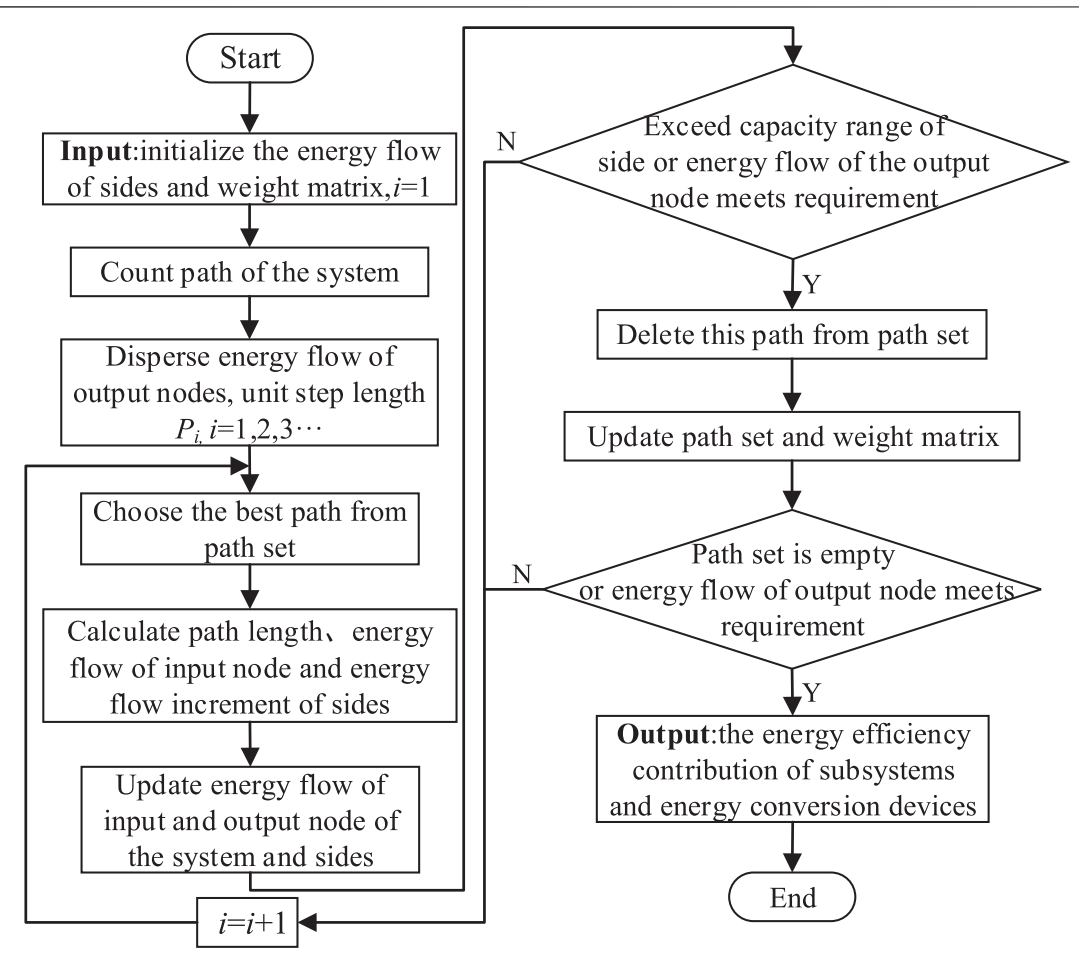

FIGURE 3 | Calculation steps based on the weighted directed graph.

Taking a demonstration park as an example, Phase I and Phase II projects are carried out gradually in temporal sequence, and every project has "cooling/heat/power/gas" load demand. Phase I was the first to be built, using the traditional energy supply method to meet the multiple loads demand in the area. The power load is supplied by purchased power and energy storage device like batteries, and the cooling load is supplied by refrigeration equipment and energy storage device, and the heat load is supplied by gas boiler and the gas load is provided by purchased natural gas. Phase II adopts multienergy complementarity of the IES to meet the load demand in the park. The installed capacity and efficiency of all equipment are shown in Table 1.

The data of cooling/heat/power/gas load in summer typical day are shown in Figure 4. The cooling load and natural gas load mainly come from appliance load. The heat load mainly includes drying technology load and appliance load. Power load includes production load and appliance load.

\section{Results of the Energy Efficiency Contribution of the Park}

Firstly, it is assumed that the configured capacity of the CHP unit is equal to its operating power, the energy efficiency contribution of the park is calculated by using the energy efficiency contribution equation, and the evaluation period is $24 \mathrm{~h}$. The data of Phase II are shown in Figure 5.

As can be seen from Figure 5, the power subsystem has the highest energy efficiency contribution in the construction of the
IES, followed by the natural gas subsystem. While the heat subsystem and the cooling subsystem have a relatively low energy efficiency contribution. The power subsystem has the highest energy efficiency contribution, because the park has very high load demand for power energy and needs to purchase power from the external grid. Moreover, the power subsystem contacts with other subsystems frequently. Otherwise, the energy efficiency contribution of the power subsystem is lower than that of the natural gas subsystem at points $9,14,15,16$, and 17 . The decrease in purchased power and the increase in demand for natural gas during these time periods are the main reasons. This leads to the increase in the energy efficiency contribution of the natural gas subsystem. Natural gas, as a common primary energy source, has a low load demand, but it is an important input energy source for energy conversion equipment, which needs to be purchased from the external gas grid. Therefore, it has a high energy efficiency contribution. Although heat and cooling subsystems have a certain load demand, they are supplied by the park itself. Therefore, the energy efficiency contribution is relatively small. The heat subsystem interacts with the other subsystems frequently; therefore, the energy efficiency contribution is higher than that of the cooling subsystem.

Figures 6-9 give the energy efficiency contribution comparison of two construction areas. The energy efficiency contribution of the subsystems in Phase II is higher than that in Phase I. It shows that the manner of energy supply in the IES is superior to the traditional manner. The results also prove the validity of the calculation method of energy efficiency contribution. 
TABLE 1 | Parameter of regional energy devices.

\begin{tabular}{|c|c|c|c|}
\hline \multirow[t]{2}{*}{ Device } & \multicolumn{2}{|c|}{ Installed capacity of the energy equipment/MW } & \multirow{2}{*}{$\begin{array}{c}\text { Equipment } \\
\text { conversion efficiency }\end{array}$} \\
\hline & Phase I & Phase II & \\
\hline CHP unit & 0 & 4.0 & $\begin{array}{l}\text { Power generation: } 0.35 \\
\text { Thermal generation: } 0.4\end{array}$ \\
\hline Gas boiler & 12.5 & 5.5 & 0.905 \\
\hline Power boiler & 0 & 5.7 & $\lambda_{\text {cop }(e-h)}=4$ \\
\hline Absorption chiller & 0 & 4.0 & 1.28 \\
\hline Refrigeration equipment & 21.0 & 16.5 & $\lambda_{c o p(e-c)}=4.5$ \\
\hline$P 2 G$ & 0 & 3.0 & 0.56 \\
\hline Battery & 5.0 & 5.0 & 0.9 \\
\hline Cooling storage device & 12.0 & 12.0 & 0.98 \\
\hline
\end{tabular}

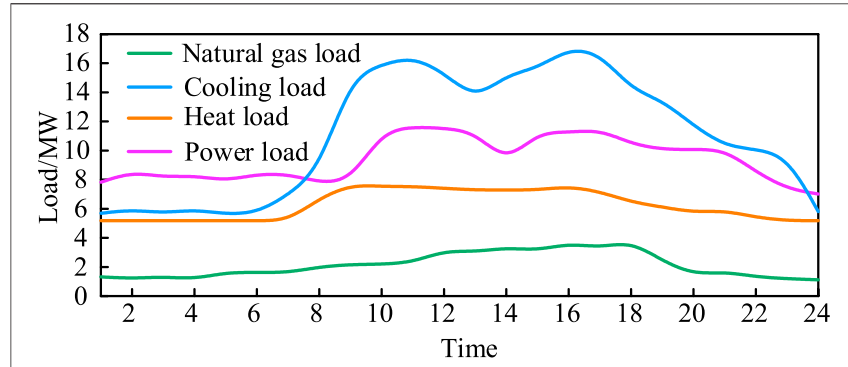

FIGURE 4 | Curves of power/heat/gas/cooling load in summer typical day.

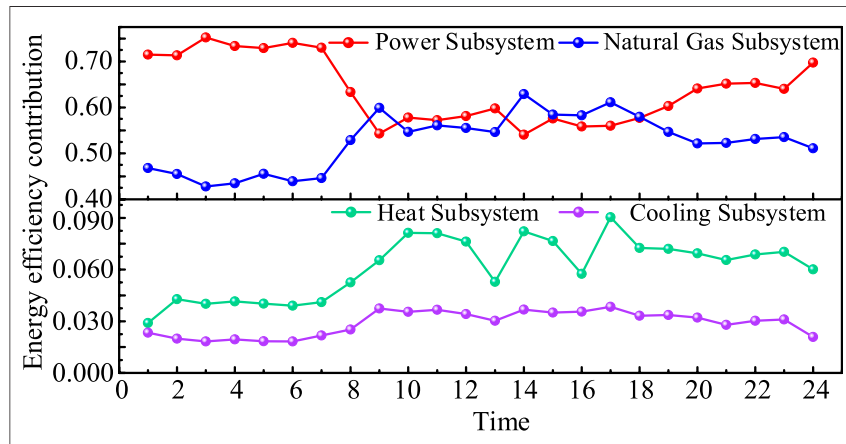

FIGURE 5 | Curves of energy efficiency contribution in Phase II.

\section{Impact of the Energy Storage Scheme on the Energy Efficiency Contribution}

Energy storage device is an important device in the energy subsystem, which can realize peak cutting and valley filling. Therefore, it plays an important role in the economic operation of the IES. However, there is energy loss in the operation process of the energy storage device, which will influence the energy efficiency contribution value of the energy subsystem. This subsection takes Phase II as an example and includes the battery and the cooling storage device. This content analyzes the energy efficiency contribution of different configuration schemes.

Four configuration schemes are developed according to the requirements, where scheme 1 indicates that the energy

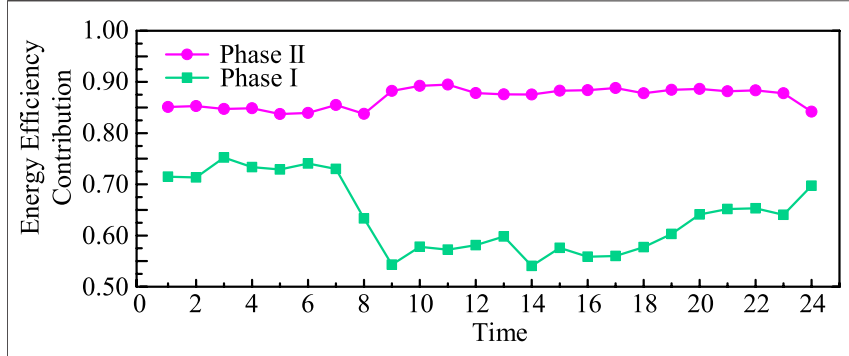

FIGURE 6 | The comparison of the power subsystem.

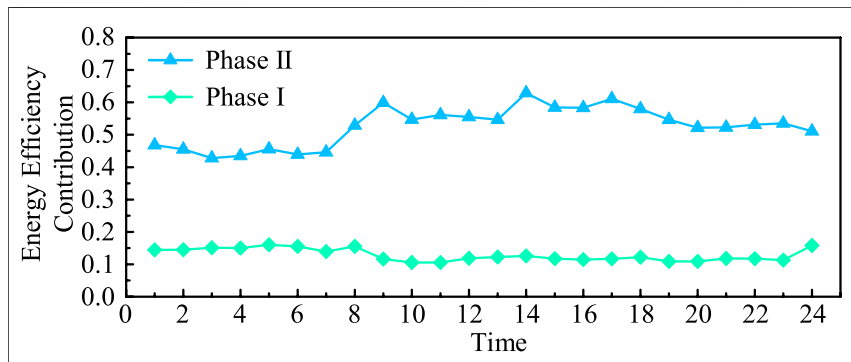

FIGURE 7 | The comparison of the natural gas subsystem.

storage devices are all in operation, scheme 2 indicates that only the cooling storage device is in operation, scheme 3 indicates that only the battery is in operation, and scheme 4 indicates that none of the energy storage devices are in operation. The results are shown in Figure 10. Considering the energy loss of the energy storage devices, the introduction of energy storage devices will reduce the energy efficiency contribution of the energy subsystem in the park. While the operation cost of the system will decrease because of the reasonable invocation of energy storage devices. The cost of each configuration scheme is shown in Table 2 .

Although the introduction of energy storage device will lead to energy loss and reduce the energy efficiency contribution of subsystems, they not only reduce the operation cost of the park, but also realize peak cutting, and valley filling and reduce the 


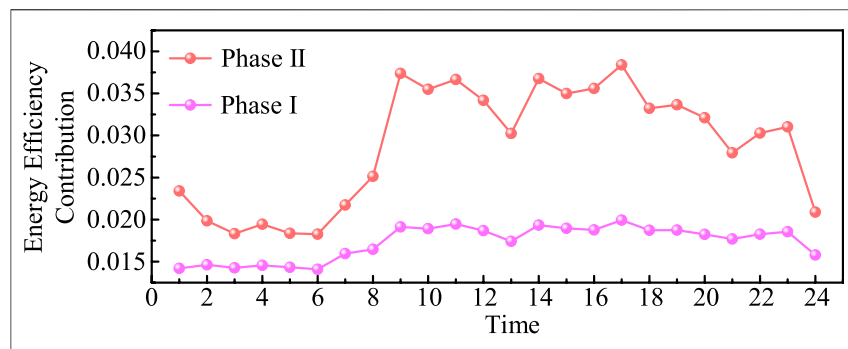

FIGURE 8 | The comparison of the cooling subsystem.



FIGURE 9 | The comparison of the heat subsystem.

frequent start-up and shut-down of units. Therefore, a comprehensive planning of the park construction is required.

\section{CONCLUSION}

In this study, a method for calculating the energy efficiency contribution of the IES is proposed and is simulated via the case study. Through the calculation and analysis of the energy efficiency contribution of energy subsystems and energy conversion devices, some conclusions can be drawn as follows:

1) Due to the complementarity of multiple energy sources, this article proposes the computational expressions of system energy efficiency and energy conversion device efficiency. According to these expressions, energy efficiency contribution models of the energy subsystems are inferred to analyze the influence of energy subsystem on the comprehensive energy efficiency. Hence, all subsystems can be classified as adjustment basis when system energy efficiency decreases.

2) The operation cost of the IES will increase when the energy storage device is put in operation, but it will improve the energy efficiency contribution of the system. Therefore, the construction of the park needs to be planned rationally and takes into account both the contribution index and the economic index.

The research results in this article present new content on the basis of traditional energy efficiency assessment. As an important index for the rational planning and operational

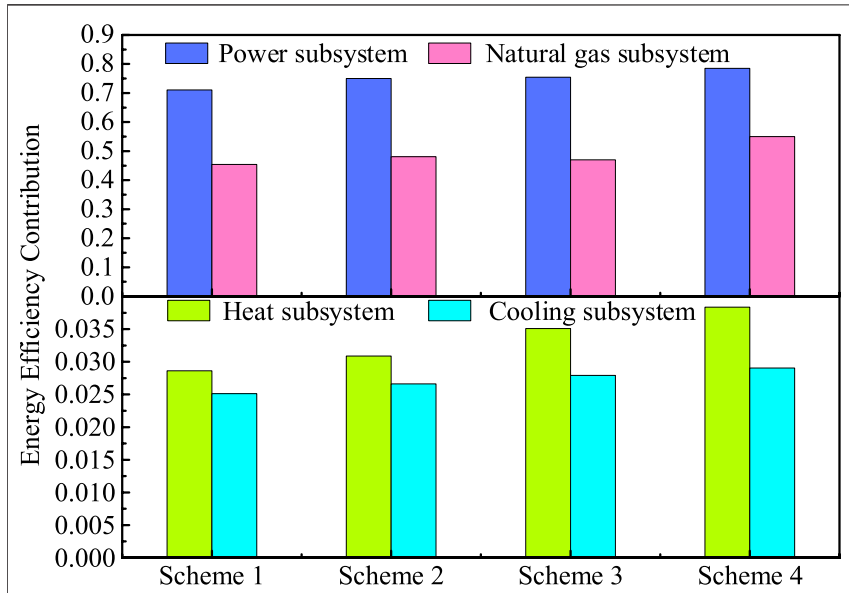

FIGURE 10 | The comparison of energy efficiency contribution under four configuration schemes.

TABLE 2 | Operation cost under different configuration schemes.

\begin{tabular}{lcccc}
\hline Scheme & $\mathbf{1}$ & $\mathbf{2}$ & $\mathbf{3}$ & $\mathbf{4}$ \\
\hline Operation Cost/ & $24,924.4$ & $27,672.6$ & $24,925.4$ & $30,427.44$
\end{tabular}

adjustment of the IES, the energy efficiency contribution index can help system operator to choose a reasonable operation plan under different energy supply methods, configuration schemes and operation methods in the system, which has guiding significance for improving the comprehensive energy efficiency of the system.

\section{DATA AVAILABILITY STATEMENT}

The original contributions presented in the study are included in the article/supplementary material; further inquiries can be directed to the corresponding author.

\section{AUTHOR CONTRIBUTIONS}

SY and MC were responsible for the construction of the energy efficiency indexes. SY and QZ focused on deducing the energy efficiency contribution models, and SY searched for a reasonable method to handle the models. The case study of this research was conducted by SY and QZ. The article was written by SY.

\section{FUNDING}

This work was supported by the Project of State Grid Jiangsu Electric Power Co., Ltd. (J2020115). 


\section{REFERENCES}

Abu-Rayash, A., and Dincer, I. (2020). Development of an Integrated Energy System for Smart Communities. Energy 202, 117683. doi:10.1016/j.energy.2020.117683

Alves, O., Monteiro, E., Brito, P., and Romano, P. (2016). Measurement and Classification of Energy Efficiency in HVAC Systems. Energy and Buildings 130, 408-419. doi:10.1016/j.enbuild.2016.08.070

Birol, F., and Keppler, J. H. (2000). Prices, Technology Development and the Rebound Effect. Energy Policy 28 (6), 457-469. doi:10.1016/S0301-4215(00)00020-3

Choi, J.-Y., Choi, I.-S., Ahn, G.-H., and Won, D.-J. (2018). Advanced Power Sharing Method to Improve the Energy Efficiency of Multiple Battery Energy Storages System. IEEE Trans. Smart Grid 9 (2), 1292-1300. doi:10.1109/ TSG.2016.2582842

Elmirghani, J. M. H., Klein, T., Hinton, K., Nonde, L., Lawey, A. Q., El-Gorashi, T. E. H., et al. (2018). GreenTouch GreenMeter Core Network Energy-Efficiency Improvement Measures and Optimization. J. Opt. Commun. Netw. 10 (2), A250-A269. doi:10.1364/JOCN.10.00A250

Filippini, M., and Hunt, L. C. (2016). Measuring Persistent and Transient Energy Efficiency in the US. Energy Efficiency 9 (3), 663-675. doi:10.1007/s12053-015-9388-5

Kavousian, A., and Rajagopal, R. (2014). Data-Driven Benchmarking of Building Energy Efficiency Utilizing Statistical Frontier Models. J. Comput. Civ. Eng. 28 (1), 79-88. doi:10.1061/(ASCE)CP.1943-5487.0000327

Kong, X., Sun, F., Huo, X., Li, X., and Shen, Y. (2020). Hierarchical Optimal Scheduling Method of Heat-Electricity Integrated Energy System Based on Power Internet of Things. Energy 210, 118590. doi:10.1016/j.energy.2020.118590

Li, P., Dong, B., Yu, H., Wang, C., Huo, Y., Li, S., et al. (2019). A Unified Energy Bus Based Multi-Energy Flow Modeling Method of Integrated Energy System. Energ. Proced. 159, 418-423. doi:10.1016/j.egypro.2018.12.066

Liu, J., Li, K., Liu, B., and Li, G. (2020). Improvement of the Energy Evaluation Methodology of Individual Office Building with Dynamic Energy Grading System. Sust. Cities Soc. 58, 102133. doi:10.1016/j.scs.2020.102133

Miao, B., Lin, J., Li, H., Liu, C., Li, B., Zhu, X., et al. (2020). Day-Ahead Energy Trading Strategy of Regional Integrated Energy System Considering Energy Cascade Utilization. IEEE Access 8, 138021-138035. doi:10.1109/ ACCESS.2020.3007224

Qin, C., Wang, L., Han, Z., Zhao, J., and Liu, Q. (2021). Weighted Directed Graph Based Matrix Modeling of Integrated Energy Systems. Energy 214, 118886. doi:10.1016/j.energy.2020.118886

Torres, J., Moreno-Torres, P., Navarro, G., Blanco, M., and Lafoz, M. (2018). Fast Energy Storage Systems Comparison in Terms of Energy Efficiency for a Specific Application. IEEE Access 6, 40656-40672. doi:10.1109/ ACCESS.2018.2854915
Wang, H., Zhang, R., Peng, J., Wang, G., Liu, Y., Jiang, H., et al. (2017). GPNBIinspired MOSFA for Pareto Operation Optimization of Integrated Energy System. Energ. Convers. Manag. 151, 524-537. doi:10.1016/ j.enconman.2017.09.005

Wang, J.-J., Yang, K., Xu, Z.-L., and Fu, C. (2015). Energy and Exergy Analyses of an Integrated CCHP System with Biomass Air Gasification. Appl. Energ. 142, 317-327. doi:10.1016/j.apenergy.2014.12.085

Wang, Y., Zhang, N., Kang, C., Kirschen, D. S., Yang, J., and Xia, Q. (2019). Standardized Matrix Modeling of Multiple Energy Systems. IEEE Trans. Smart Grid 10 (1), 257-270. doi:10.1109/TSG.2017.2737662

Willem, H., Lin, Y., and Lekov, A. (2017). Review of Energy Efficiency and System Performance of Residential Heat Pump Water Heaters. Energy and Buildings 143, 191-201. doi:10.1016/j.enbuild.2017.02.023

Wang, J., Zhong, H., Yang, Z., Lai, X., Xia, Q., and Kang, C. (2020). Incentive Mechanism for Clearing Energy and Reserve Markets in Multi-Area Power Systems. In IEEE Transactions on Sustainable Energy 11 (4), 2470-2482. doi:10.1109/TSTE.2019.2961780

Xue, Y. (2015). Energy Internet or Comprehensive Energy Network?. J. Mod. Power Syst. Clean. Energ. 3 (3), 297-301. doi:10.1007/s40565-015-0111-5

Zhang, T., Wang, J., Zhong, H., Li, G., Zhou, M., and Zhao, D. (2020). Soft Open Point Planning in Renewable-Dominated Distribution Grids with Building Thermal Storage. In CSEE Journal of Power and Energy Systems. doi:10.17775/ CSEEJPES.2020.03660

Zou, G., Chen, L., Liu, W., Hong, X., Zhang, G., and Zhang, Z. (2013). Measurement and Evaluation of Chinese Regional Energy Efficiency Based on Provincial Panel Data. Math. Comp. Model. 58 (5), 1000-1009. doi:10.1016/ j.mcm.2012.09.020

Conflict of Interest: Authors SY, MC and QZ were employed by the company State Grid Jiangsu Electric Power Co. LTD.

Publisher's Note: All claims expressed in this article are solely those of the authors and do not necessarily represent those of their affiliated organizations, or those of the publisher, the editors, and the reviewers. Any product that may be evaluated in this article, or claim that may be made by its manufacturer, is not guaranteed or endorsed by the publisher.

Copyright $\odot 2021$ Yang, Chen and Zuo. This is an open-access article distributed under the terms of the Creative Commons Attribution License (CC BY). The use, distribution or reproduction in other forums is permitted, provided the original author(s) and the copyright owner(s) are credited and that the original publication in this journal is cited, in accordance with accepted academic practice. No use, distribution or reproduction is permitted which does not comply with these terms. 